\title{
Olfactory identification dysfunction, aggression and impulsivity in war veterans with post-traumatic stress disorder
}

\author{
J. F. Dileo ${ }^{1}$, W. J. Brewer ${ }^{2 *}$, M. Hopwood ${ }^{3}$, V. Anderson ${ }^{4}$ and M. Creamer ${ }^{5}$ \\ ${ }^{1}$ Department of Psychology, University of Melbourne, Australia \\ ${ }^{2}$ ORYGEN Research Centre, Department of Psychiatry, University of Melbourne, Australia \\ ${ }^{3}$ Veterans Psychiatry Unit, Austin Health, University of Melbourne, Australia \\ ${ }^{4}$ Murdoch Children's Research Institute, Melbourne, Australia \\ ${ }^{5}$ Australian Centre for Posttraumatic Mental Health, University of Melbourne, Australia
}

Background. Due to neuropsychological conceptualizations of orbitoprefrontal cortex (OFC) dysfunction underpinning impulsive aggression and the incidence of such behaviour in post-traumatic stress disorder (PTSD), this study aimed to explore olfactory identification (OI) ability in war veterans with PTSD as a probe of putative OFC dysfunction; and to explore the utility of OI ability in predicting aggressive and impulsive behavior in this clinical population.

Method. Participants comprised 31 out-patient male war veterans with PTSD (mean $=58.23$ years, S.D. $=2.56$ ) recruited from a Melbourne Veterans Psychiatry Unit, and 31 healthy age- and gender-matched controls (mean=56.84 years, S.D. $=7.24$ ). All participants were assessed on clinical measures of PTSD, depression, anxiety, and alcohol misuse; olfactory identification; neurocognitive measures of dorsolateral prefrontal, lateral prefrontal and mesial temporal functioning; and self-report measures of aggression and impulsivity.

Results. War veterans with PTSD exhibited significant OI deficits (OIDs) compared to controls, despite uncompromised performance on cognitive measures. OIDs remained after covaring for IQ, anxiety, depression and alcohol misuse, and were significant predictors of aggression and impulsivity.

Conclusions. This research contributes to emerging evidence of orbitoprefrontal dysfunction in the pathophysiology underlying PTSD. This is the first study to report OIDs as a predictor of aggression and impulsivity in this clinical population. It prompts further exploration of the potential diagnostic utility of OIDs in the assessment of PTSD. Such measures may help delineate the clinical complexity of PTSD, and support more targeted interventions for individuals with a greater susceptibility to aggressive and impulsive behaviors.

Received 30 October 2006; Revised 19 March 2007; Accepted 6 July 2007; First published online 1 October 2007

Key words: Aggression, impulsivity, olfactory identification, post-traumatic stress disorder.

\section{Introduction}

Post-traumatic stress disorder (PTSD) is a debilitating condition characterized by symptoms of avoidance, hyperarousal and intrusive memories, affecting $10-20 \%$ of the $60 \%$ of adults that experience a severe trauma (Kessler et al. 1995). Additional to the symptomatic triad of PTSD are anti-social personality outcomes such as increased aggression, anger, impulsivity and suicidal thoughts and behavior (Chemtob et al. 1994; Joseph et al. 1997; Begic \& JokicBegic, 2001; Kotler et al. 2001). This condition is often co-morbid with alcohol abuse, mood disorders,

* Address for correspondence: Associate Professor W. Brewer, ORYGEN Research Centre (Locked bag 10), Parkville, Victoria, 3052, Australia.

(Email:w.brewer@unimelb.edu.au) personality disorders and persistent health problems, thereby posing a significant challenge in diagnosis and treatment (O'Donnell et al. 2004; Turner et al. 2005). Elucidating the neurobiology of PTSD and more specific patterns in course and presentation will help improve the efficacy of targeted therapeutic interventions.

Current neurobiological models of PTSD implicate a number of interdependent neurobiological systems and structures including the executive role in emotion modulation and conditioning processes of the orbitoprefrontal cortex (OFC; Vermetten \& Bremner, 2002). In addition, OFC dysfunction has been implicated in the pathophysiology of aggressive and impulsive behavior (Pietrini et al. 2000; Best et al. 2002; Blair, 2004) such as that seen in individuals with PTSD (Chemtob et al. 1994; Begic \& Jokic-Begic, 2001). Innervated with 
the basolateral amygdala, the OFC plays a central role in emotion modulation, in processes that amplify, attenuate, or maintain the emotional response (Seguin, 2004). Aggressive and impulsive behaviors are believed to be the result of failure of medial prefrontal structures in asserting executive control over amplified amygdala responsiveness, and a general failure in integrating these cues and responses within a social context to inform appropriate social behavior (Davidson et al. 2000). There has been limited research of OFC functioning in individuals with PTSD.

Due to the challenge of delineating the neurocorrelates of impairment in psychiatric conditions, olfactory identification deficits (OIDs) are being increasingly employed as a measure of OFC integrity (Martzke et al. 1997; Brewer et al. 2006). The OFC mediates odor identification as found via lesion and neuroimaging studies (Martzke et al. 1997). After odor detection is facilitated in the piriform cortex of the limbic system, olfactory projections innervate the OFC where identification occurs (Zatorre et al. 1992). As the limbic-prefrontal neural pathways for olfactory identification (OI) parallel circuits involved in emotion modulation, OIDs have been strongly associated with impaired inhibition of affect, delusions, and maladaptive behavior (Martzke et al. 1997; Brewer et al. 2006). Although odors are recognized as strong elicitors of traumatic memories (Kline \& Rausch, 1985; Vermetten \& Bremner, 2003), only two recent experimental studies (Vasterling et al. 2000, 2003) have reported OIDs in clinical populations with PTSD.

Vasterling et al. (2000) found that Vietnam war veterans with PTSD exhibited significant OIDs relative to veterans without PTSD, and to matched controls. Furthermore, these OIDs occurred in the presence of relatively intact performance on measures of dorsolateral and mesial temporal functioning, suggesting localized OFC impairment. In their subsequent study, Vasterling et al. (2003) explored possible neurotoxic effects of war zone exposure in Gulf War veterans. Results suggested that war zone participation did not have an effect on OI or on cognitive performance. Whilst these studies implicate the role of OFC pathophysiology underpinning PTSD neither study controlled for measures of psychiatric co-morbidity and neither explored the relationship between OIDs and behavioral manifestations of PTSD.

Due to the olfactory system's unique localization of affect sub-processes to neocortical and subcortical regions (Hamann, 2003), OI tasks are useful for understanding the neuropsychology of PTSD (Hamann, 2003; Brewer et al. 2006). Emotion-focused research has focused on two primary dimensions, pleasantness and intensity. The unique engineering of the human olfactory system is ideal for operationalizing these constructs (Hamann, 2003). Processing of intensity ratings of odors recruits amygdala, entorhinal and piriform cortices regardless of pleasantness (Zatorre et al. 2000; Anderson et al. 2003; Rolls et al. 2003), while processing the pleasantness of odors implicates the OFC (de Araujo et al. 2003). Furthermore, animal study findings highlight the role of the OFC in olfactory discrimination learning and aversive conditioning based on pleasantness, consolidating the notion that this region is specialized for adaptive conditioning (Critchley \& Rolls, 1996; Rolls, 2000). The relevance of OIDs in individuals with PTSD to outcomes such as aggression and impulsivity remains unclear.

This study aims to explore OI as a proxy measure of OFC function in a PTSD cohort and to investigate the relationships between OIDs and aggressive and impulsive behavior in war veterans with PTSD. Consistent with Vasterling et al. (2000) PTSD patients are expected to demonstrate greater OIDs compared to controls. It is also hypothesized that OIDs will be a significant predictor of impulsivity and aggression in this clinical group. Regarding the OFC's specialized role in processing the pleasantness of odors (Hamann, 2003), this study also aimed to examine the influence of odor pleasantness on odor identification.

\section{Method}

\section{Participants}

Participants were 31 male Vietnam war veterans $($ mean $=58.23$, s.D. $=2.56)$ with a primary diagnosis of PTSD recruited from an out-patients veterans psychiatry unit. As gender variation has been found in OI performance (Doty et al. 1984), only male participants were recruited. Due to the high prevalence of comorbidity with anxiety, depression, and alcohol misuse, patients with such diagnoses were not excluded but data on the same was collected. The control group comprised 31 healthy age-matched males $($ mean $=56.84$, s.D. $=7.24)$ with no history of mental health complications, recruited through exservice organizations and by word of mouth.

All participants were required to have an adequate command of English based on the National Adult Reading Test (NART; Nelson \& O'Donnell, 1978) premorbid IQ score being $>70$, and were excluded if they reported: (a) a documented organic brain impairment or epilepsy; $(b)$ history of a head injury that resulted in a loss of consciousness for > $30 \mathrm{~min}$; (c) current medical condition (viral or other) that impaired respiratory processes; (d) a history of nasal trauma; (e) documented hearing or visual impairment; and $(f)$ thyroid problems. Based on 
these criteria, three individuals were excluded due to previous head injury, one was excluded due to a premorbid IQ score $<70$ on the NART, and two participants were excluded due to severe smell detection problems.

All but one member of the PTSD group were taking some form of medication at the time of assessment, and one control group participant was taking medication for a physical ailment. Medication for a physical ailment was being taken by $73 \%$ of the PTSD group, $67 \%$ were taking antidepressants, $21 \%$ were taking anxiolytics, $12 \%$ were taking mood stabilizers and $12 \%$ were taking medication for sleep problems. Whilst the influence of medication on smell detection may be problematic, the effect on identification once the stimulus is detected is less controversial. Analysis of medicated and unmedicated participants was conducted across OI, neurocognitive and personality measures. A within-subjects ANCOVA covarying for IQ found no significant effects of any medication on any neurocognitive or personality measure, except for anxiolytics on self-reported impulsivity $[F(1,30)=15.17, p<0.01]$. No significant difference in smoking behavior or not was found between the control and PTSD groups for current $\left(\chi^{2}=0.86\right.$, $p=0.35)$ and previous smoking history $\left(\chi^{2}=1.59\right.$, $p=0.21$.

\section{Measures}

\section{Clinical measures}

All participants were assessed for PTSD, anxiety, depression and alcohol misuse. PTSD symptoms and severity were assessed via the Posttraumatic Stress Disorder Checklist Military (PCL-M), a 17-item selfreport questionnaire (Weathers et al. 1993). Anxiety and depression were measured via the Hospital Anxiety and Depression Scale (HADS), a 14-item selfreport measure with severity scores for anxiety and depression (Zigmond \& Snaith, 1983). Alcohol use was assessed via the Alcohol Use Disorders Identification Test (AUDIT), a 10-item questionnaire that measures alcohol consumption, dependence and problems relating to alcohol use (Babor et al. 1989).

\section{Olfactory identification}

OI was assessed via the University of Pennsylvania Smell Identification Test (UPSIT; see Doty et al. 1984 for review), a 40-item suprathreshold 'scratch and sniff' multiple-choice test. In order to assess possible laterality effects, participants were assessed on 20 items via the left nostril, and 20 items via the right nostril by blocking one nostril. The order of nostril presentation was counterbalanced across participants. To account for subjectivity in the hedonic response to each of the 40 odors, normative data from Doty et al. (1984) was employed to categorize the items into 17 pleasant, 10 unpleasant, and 13 neutral odors. Due to their being unequal items in each category and between subsets of 20 items for each nostril, scores for all UPSIT subscores were converted to a percentage correct. As the UPSIT assesses odor identification and assumes detection capacity, participants were expressly asked to inform the researcher if they could not detect an odor. In these instances, the panel was scratched until detection was achieved. Due to language and cultural differences between North America and Australia, a 2-point correction was made to total UPSIT scores (Mackay-Sim \& Doty, 2001).

\section{Cognitive functioning}

A battery of verbal and non-verbal tasks, sensitive to prefrontal and medial temporal functioning was selected. Verbal fluency was measured via the Controlled Oral Word Association Task (COWAT; Borkowoski et al. 1967). New word learning and verbal memory was assessed via the Verbal Paired Associates Task (VPA; Wechsler, 1987). Sustained attention and psychomotor inhibition were measured via the Go/ No-Go task (GNG; Luria, 1969). A composite score was created by regressing errors of commission or false alarms and reaction time for this task $[F(1,60)=9.56, p<0.01]$. The Rey-Osterrieth Complex Figure (RCF; Rey, 1941) copy and delayed recall task was administered as a measure of visuospatial construction, organization and recall, as scored by Taylor's (1959) scoring system for copy and recall, and Bennett-Levy's (1984) strategy scoring system for measuring planning and organization. The NART (Nelson \& O'Donnell, 1978) was administered as a measure of premorbid IQ.

\section{Aggression and impulsivity}

Self-report personality measures were selected to assess aggression and impulsivity. The Buss and Perry Aggression Questionnaire (BPAQ) is a 29-item self-report questionnaire, rating each individual on measures of physical aggression, verbal aggression, anger, hostility and overall aggression (Buss \& Perry, 1992). Eysenck's Impulsivity Questionnaire i7 (i7) is a 54-item self-report questionnaire employed to profile each individual on measures of impulsivity, venturesomeness, and empathy (Eysenck et al. 1985).

\section{Procedure}

Following informed consent, each assessment commenced with an informal interview to capture data on medication, medical history, smoking, and current 
Table 1. Comparison of war veterans with PTSD and age- and gender-matched control group on clinical measures for PTSD, anxiety, depression, and alcohol misuse

\begin{tabular}{|c|c|c|c|c|c|c|}
\hline & \multirow{2}{*}{$\begin{array}{l}\text { Clinical } \\
\text { level }\end{array}$} & \multicolumn{2}{|c|}{ PTSD group $(n=31)$} & \multicolumn{2}{|c|}{ Control group $(n=31)$} & \multirow{2}{*}{$\begin{array}{l}\text { Between-group } \\
\text { effect size } \\
\omega^{2}\end{array}$} \\
\hline & & Mean & S.D. & Mean & S.D. & \\
\hline Intrusive memories & & 19.23 & 5.95 & 9.04 & 3.11 & 0.53 \\
\hline Arousal & & 21.23 & 3.89 & 8.41 & 2.97 & 0.77 \\
\hline Avoidance & & 20.77 & 3.70 & 8.49 & 3.33 & 0.75 \\
\hline PTSD total & 50 & 61.23 & 9.57 & 25.94 & 8.35 & 0.79 \\
\hline Anxiety & 11 & 12.35 & 2.73 & 3.51 & 3.26 & 0.68 \\
\hline Depression & 11 & 11.97 & 4.01 & 2.27 & 2.53 & 0.67 \\
\hline Alcohol & 8 & 3.53 & 2.35 & 2.28 & 1.15 & 0.09 \\
\hline
\end{tabular}

mental health to determine eligibility. All participants were then guided consistently through the clinical measures (PCL-M, HADS, and AUDIT), followed by the NART, UPSIT, RCF copy task, BPAQ, COWAT, VPA, i7, RCF recall, and GNG tasks.

\section{Data analysis}

Statistical analyses were conducted using the Statistical Package for the Social Sciences, version 13 (SPSS Inc., Chicago, IL, USA). Following preliminary analysis of data to ensure statistical assumptions were met, a square root transformation was performed on GNG and AUDIT scores to address negative skewness. Initial between-group $t$ tests were used to explore differences on continuous demographic measures, and $\chi^{2}$ tests were employed for non-parametric data. Due to significant between-group differences on premorbid IQ, between-group ANCOVAs were conducted covarying for IQ for all between-group analysis. The general linear model (GLM) in SPSS was used calculating type III sums of squares, and the weighted marginal means were assessed. Due to the limitations of the GLM in SPSS to account for covariates during within-subject analysis (Page et al. 2003), mixed factorial ANOVAs were used for these analyses. Omega squared $\left(\omega^{2}\right)$ was calculated for each test to produce unbiased effect sizes (Cohen, 1988). Pearson's product moment correlations were conducted for a preliminary analysis of the relationship between OI and self-report measures of aggression and impulsivity. Data reduction was conducted via principle components analysis for total aggression and impulsivity scores to create a composite score. These scores were found to share $79.2 \%$ common variance. Linear regression was used to explore the predictive utility of OI deficits in relation to this composite 'aggression/impulsivity' score. To counter the effect of outliers, all scores were standardized and outliers were reduced to 2 S.D. above or below the mean.

\section{Results}

\section{Clinical measures}

The PTSD group scored significantly higher on all PTSD symptom clusters and overall $[F(1,60)=239.20$, $p<0.01]$, and also recorded higher scores for anxiety $[F(1,60)=134.27, \quad p<0.01]$, depression $[F(1,60)=$ $129.61, p<0.01]$, and alcohol misuse $[F(1,60)=7.04$, $p=0.01]$, with large effect sizes for all except alcohol misuse (see Table 1).

\section{Cognitive functioning}

A between-group ANOVA found significantly higher IQ in the control group compared to the clinical group. ANCOVAs covaring for IQ found that the homogeneity of regression assumption was violated for COWAT total and RCF copy, recall and strategy tasks. Subsequent ANOVAs found no significant between-group differences on these tasks (see Table 2). For other neurocognitive tasks, ANCOVAs covarying for estimated IQ found no significant differences between groups on mistakes, repetitions or disinhibition on the COWAT; learning easy, difficult and overall pairs of words on the VPA; or false alarms and reaction time on the GNG task.

\section{Olfactory identification}

Between-group ANOVAs comparing mean UPSIT scores as a percentage of total scores found a significant disadvantage in the PTSD cohort relative to control participants at $p=0.01$ on total UPSIT performance $\left[F(1,60)=34.95, p<0.01, \omega^{2}=0.35\right]$, left nostril $\left[F(1,60)=24.83, p<0.01, \omega^{2}=0.28\right]$ and right nostril performance $\left[F(1,60)=16.75, p<0.01, \omega^{2}=\right.$ 0.20], for pleasant odors $[F(1,60)=31.72, p<0.01$, $\left.\omega^{2}=0.33\right]$ and for unpleasant odors $[F(1,60)=16.80$, $p<0.01, \omega^{2}=0.20$; see Fig. 1). No significant betweengroup differences were found for identifying neutral 
Table 2. Comparison of the performance of the PTSD and control groups on measures of neurocognitive functioning found no significant differences, except on the NART

\begin{tabular}{|c|c|c|c|c|c|c|c|c|}
\hline & & \multicolumn{2}{|c|}{ PTSD group } & \multicolumn{2}{|c|}{ Control group } & \multirow[b]{2}{*}{$F$} & \multirow[b]{2}{*}{ Sig. } & \multirow[b]{2}{*}{$\omega^{2}$} \\
\hline & & Mean & S.D. & Mean & S.D. & & & \\
\hline \multirow[t]{2}{*}{ NART } & Total score ${ }^{\mathrm{a}}$ & 30.13 & 8.64 & 39.65 & 5.51 & 26.75 & 0.00 & 0.29 \\
\hline & Estimated $\mathrm{IQ}^{\mathrm{a}}$ & 109.25 & 8.32 & 124.79 & 6.46 & 67.54 & 0.00 & 0.52 \\
\hline \multirow[t]{3}{*}{ COWAT } & Total words ${ }^{\mathrm{b}}$ & 36.88 & 12.69 & 42.41 & 14.24 & 2.60 & 0.11 & 0.03 \\
\hline & Mistakes & 0.89 & 1.33 & 0.70 & 1.01 & 0.07 & 0.79 & -0.02 \\
\hline & Repetitions & 0.76 & 1.08 & 0.80 & 1.25 & 0.90 & 0.35 & 0.00 \\
\hline \multirow[t]{3}{*}{ VPA } & Easy pairs & 10.41 & 2.14 & 10.16 & 1.61 & 0.46 & 0.50 & -0.01 \\
\hline & Difficult pairs & 4.23 & 2.88 & 5.13 & 2.56 & 0.01 & 0.91 & -0.02 \\
\hline & Total pairs & 14.64 & 4.40 & 15.29 & 3.46 & 0.16 & 0.69 & -0.01 \\
\hline GNG & Composite score & -0.20 & 1.02 & 0.20 & 0.98 & 1.44 & 0.24 & 0.02 \\
\hline \multirow[t]{3}{*}{$\mathrm{RCF}$} & Copy $^{b}$ & 22.44 & 5.39 & 22.84 & 4.93 & 0.09 & 0.76 & -0.01 \\
\hline & Recall $^{\mathrm{b}}$ & 12.40 & 5.57 & 13.32 & 4.66 & 0.49 & 0.49 & -0.01 \\
\hline & Strategy ${ }^{b}$ & 25.04 & 7.52 & 25.16 & 6.92 & 0.00 & 0.95 & -0.02 \\
\hline
\end{tabular}

NART, National Adult Reading Test; COWAT, Controlled Oral Word Association Task; VPA, Verbal Paired Associates Task; GNG, Go/No-Go task; RCF, Rey-Osterrieth Complex Figure.

${ }^{a}$ ANOVA was used to assess between-group differences on this task.

${ }^{b}$ Due to violations of the homogeneity of regression assumption for ANCOVA covarying for estimated IQ, ANOVA was used to assess between-group differences on these tasks.

odors $\left[F(1,60)=2.00, p=0.16, \omega^{2}=0.02\right]$. Between differences remained significant at the $p=0.01$ level when tested via ANCOVA, covarying for IQ, and self-reported anxiety, depression and alcohol use, however, effect sizes ranging from $\omega^{2}=0.04-0.11$ were smaller.

A mixed ANCOVA (covarying for estimated IQ) using nostril as the within-subjects variable found no significant difference between left and right nostril identification $[F(1,59)=0.39, p=0.54]$, or interaction between nostril and group $[F(1,59)=0.10, p=0.75]$. A mixed ANCOVA (covarying for estimated IQ) using odor type as the within-subjects variable found a significant interaction between odor type and group $[F(1,59)=4.03, p=0.05]$, where participants with PTSD identified neutral odors better than pleasant/unpleasant odors and the control group recorded the reverse pattern. $\chi^{2}$ analyses revealed that the PTSD group performed significantly worse than the control group in identifying nine odors at $p<0.05$ : the PTSD group were particularly vulnerable in identifying turpentine $\left(\chi^{2}=12.45, p<0.01\right)$, followed by smoke $\left(\chi^{2}=11.14, p<0.01\right)$, banana $\left(\chi^{2}=7.76\right.$, $p=0.01)$, lemon $\left(\chi^{2}=7.70, p=0.01\right)$, chocolate $\left(\chi^{2}=6.72\right.$, $p=0.01)$, pineapple $\left(\chi^{2}=5.81, p=0.02\right)$, strawberry $\left(\chi^{2}=4.24, p=0.04\right)$, lilac $\left(\chi^{2}=4.22, p=0.04\right)$, and gasoline $\left(\chi^{2}=4.17, p=0.04\right)$. These nine odors contributed to $62 \%$ of the between-group variance observed on the UPSIT.

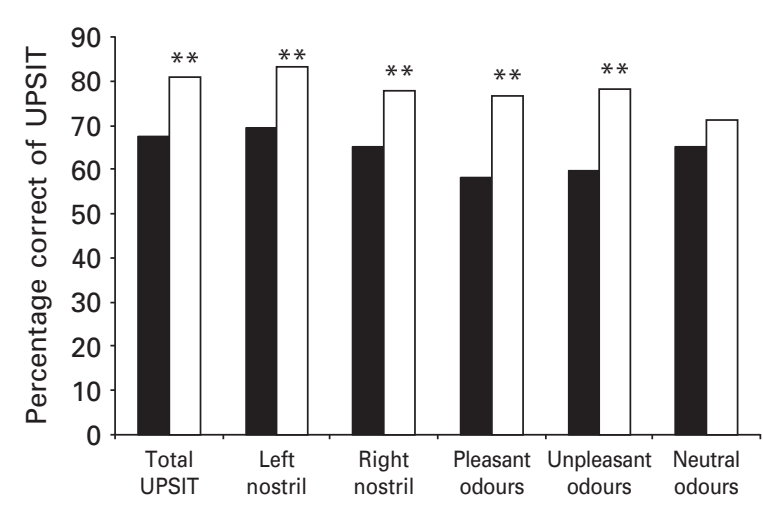

Fig. 1. Comparison of war veterans with PTSD $(\boldsymbol{\square})$ and ageand gender-matched controls $(\square)$ on the University of Pennsylvania Smell Identification Test (UPSIT) $\left({ }^{* *} p<0.01\right)$.

\section{Personality measures}

Between-group ANCOVAs covarying for estimated IQ found that the PTSD group self- reported significantly higher levels of physical aggression $\left[F(1,60)=58.21, p<0.01, \omega^{2}=0.24\right]$, verbal aggression $\left[F(1,60)=18.11, p<0.01, \omega^{2}=0.17\right]$, hostility $[F(1,60)=$ 51.64, $\left.p<0.01, \omega^{2}=0.16\right]$, anger $[F(1,60)=107.72$, $\left.p<0.01, \omega^{2}=0.29\right]$, and impulsivity $[F(1,60)=27.51$, $\left.p<0.01, \omega^{2}=0.09\right]$ than controls, reflecting effect sizes approaching small to moderate. No significant between-group differences were found on 
Table 3. Pearson product-moment correlations ( $r$ ) between olfactory identification deficits and Buss and Perry Aggression Questionnaire and $i 7$ subscales

\begin{tabular}{|c|c|c|c|c|c|c|c|c|}
\hline & $\begin{array}{l}\text { Physical } \\
\text { Aggression }\end{array}$ & $\begin{array}{l}\text { Verbal } \\
\text { Aggression }\end{array}$ & Hostility & Anger & $\begin{array}{l}\text { Total } \\
\text { Aggression }\end{array}$ & Impulsivity & $\begin{array}{l}\text { Venturesome- } \\
\text { ness }\end{array}$ & Empathy \\
\hline UPSIT total & $-0.32^{*}$ & $-0.25^{*}$ & $-0.31^{*}$ & $-0.45^{* *}$ & $-0.39^{* *}$ & $-0.31^{*}$ & 0.10 & -0.05 \\
\hline Pleasant odors & $-0.35^{* *}$ & $-0.32^{*}$ & $-0.41^{* *}$ & $-0.55^{* *}$ & $-0.46^{* *}$ & $-0.37^{* *}$ & 0.23 & -0.05 \\
\hline Unpleasant odors & -0.22 & -0.10 & -0.12 & -0.21 & -0.19 & -0.18 & -0.06 & 0.00 \\
\hline Neutral odors & -0.07 & -0.02 & -0.10 & -0.11 & -0.09 & -0.07 & 0.02 & -0.07 \\
\hline
\end{tabular}

UPSIT, University of Pennsylvania Smell Identification Test.

* Correlation is significant at the 0.05 level (two-tailed).

** Correlation is significant at the 0.01 level (two-tailed).

venturesomeness $\left[F(1,60)=3.14, p=0.08, \omega^{2}=0.01\right]$ or empathy scales $\left[F(1,60)=0.31, p=0.58, \omega^{2}=0.01\right]$.

Significant Pearson product-moment correlations were found between total UPSIT scores and physical aggression, verbal aggression, hostility, anger, total aggression, and impulsivity, but not venturesomeness or empathy (Table 3). These correlations were stronger with pleasant odor identification, but weaker and not significant for unpleasant or neutral odors.

Linear regression found that total UPSIT scores were a significant predictor of the composite aggression/impulsivity scores $\left[r^{2}=0.16: F(1,60)=11.08\right.$, $p<0.01$; see Fig. 2].

\section{Discussion}

The current study contributes to emerging evidence of OIDs in combat veterans with PTSD (Vasterling et al. 2000, 2003), where those with this primary diagnosis performed significantly worse than age- and gendermatched normal controls. Furthermore, these deficits occurred in the context of relatively unimpaired performance on neurocognitive tasks that are sensitive to dorsolateral prefrontal, medial prefrontal, and medial temporal functioning. In addition, this study is the first to report OIDs as a predictor of aggression and impulsivity, which were significantly higher in the PTSD group. Moreover, a significant double dissociation in OI performance by odor type was found, where control participants exhibited stronger identification of pleasant and unpleasant than neutral odors, and PTSD participants demonstrated the reverse pattern. Together with previous literature suggesting the OID performance may be a proxy measure of OFC integrity (Zatorre et al. 1992; Brewer et al. 2006), these findings support the hypothesis of underlying OFC dysfunction in the pathophysiology of PTSD, and suggest that OIDs may have diagnostic utility in predicting aggression and impulsivity in this clinical population.

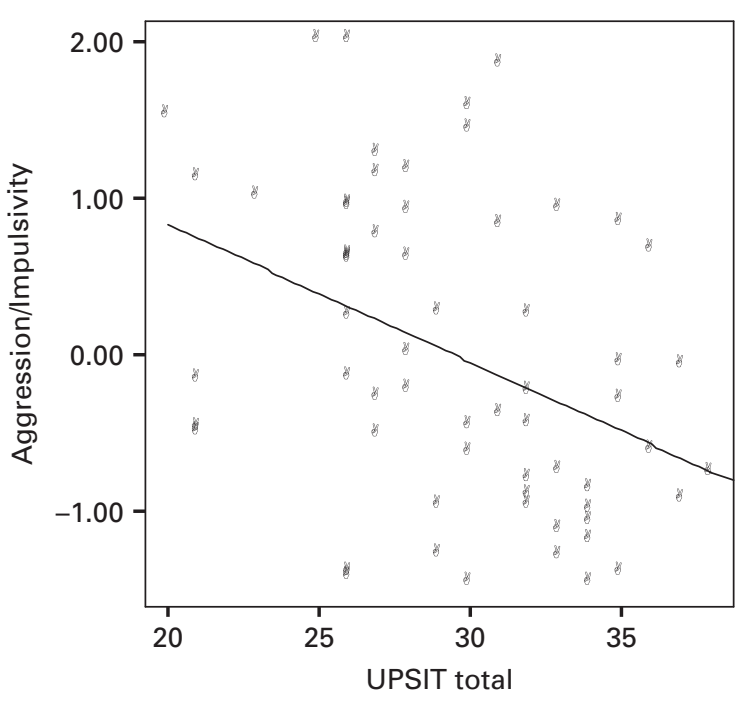

Fig. 2. Negative linear relationship between total University of Pennsylvania Smell Identification Test (UPSIT) scores and the Aggression/Impulsivity composite score. Aggression/ Impulsivity $=2.60+-0.90 \times \operatorname{UPSIT}\left(R^{2}=0.16\right)$.

Consistent with Vasterling et al. (2000, 2003), the OIDs in the PTSD group ranged from moderate to severe microsmia. These deficits could not be accounted for by smoking or medication and they remained after controlling for co-morbid anxiety, depression, alcohol misuse and estimated IQ. No between-group differences on cognitive tasks relying upon intact dorsolateral prefrontal, ventral prefrontal, and medial temporal functioning were found, suggesting relatively more localized OFC dysfunction in the pathophysiology underpinning PTSD.

As previous research has also identified that processing of odor pleasantness is mediated by the OFC (Anderson et al. 2003), the particular deficit our PTSD sample had in identifying pleasant or unpleasant odors is also consistent with the putative compromise of OFC processing. With nine odors contributing to $62 \%$ of the between-group differences, and the two 
most poorly identified odors in the PTSD participants being smoke and turpentine, our findings suggest that OIDs may not be random. It is probable that many Vietnam veterans in our sample would have been exposed to odors such as smoke, turpentine, gasoline and banana that are prevalent in a tropical war zone, which may explain why our PTSD cohort apparently experienced more extreme emotional responses during the assessment that prevented access to the correct verbal lexicon for identification. Conversely, following traumatic exposure, desensitization to these odors may also have occurred to the extent that our cohort did not benefit from the affective attributions that appeared to aid OID in controls. Clearly, hedonic associations such as pleasantness are personal, specific and contextualized for each individual. However, the strong interaction effect identified in our study would suggest that affect attribution in odor identification may be an important sub-process in this psychiatric population that warrants further exploration.

As anticipated, OIDs were significant predictors of aggression and impulsivity, which were reported at significantly higher levels in the PTSD group. These findings add further evidence of putative OFC dysfunction in individuals with PTSD (Vasterling et al. 2000, 2003), reflecting both dysfunction in OFC-mediated executive control over limbic emotion responsiveness, and maladaptive social and emotional learning. Therefore, PTSD sufferers may not benefit from flexible, adaptive and reflective integration of social cues, and are therefore more potently influenced by strong, conditioned, instinctive responses particularly aggression. Our findings suggest such a disposition remains after more than 25 years in combat, consistent with the nature of conditioning. This highlights the importance of targeting such behavior in therapeutic interventions following trauma. In the context of likely neurotoxic effects of prolonged periods of extreme arousal on limbic structures, it is possible that neurobiological alterations such as subcortical atrophy may mediate these findings (Oozthuizen et al. 2005). Future research employing structural and functional imaging of limbic-prefrontal circuitry in individuals with PTSD could further our understanding of the pathophysiology of this dysfunction.

Our results also suggest that the relationship between OIDs and aggressive and impulsive behavior are strongly related to the hedonic properties of the odor, such that a negative relationship was only observed with pleasant, but not unpleasant or neutral odors. As the OFC receives a major serotonergic input (Rolls, 1998) and there was a high rate of co-morbid depression in our sample, these olfactory deficits may relate to low serotonergic activity in the OFC and insufficient positive affect to elicit recall. This hypothesis is consistent with the findings of Stanley et al. (2000), who found significantly lower concentrations of CSF 5-hydroxyindoleacetic acid (5-HIAA) in their aggressive versus non-aggressive groups of psychiatric patients. Clearly impulsive aggression can be idiosyncratic, and specific to personal experience, as is the sense of pleasantness that an individual associates with odors. However, given that no relationship was found between unpleasant and neutral odors and impulsive and aggressive behaviors, this finding cannot be overlooked. Future studies may benefit from exploring the properties of valence and intensity in odor identification in larger populations, and explore their relationship with PTSD symptoms and behavioral outcomes.

Our study has several limitations. First, the control group of age-matched males was recruited from the community, where recruiting war veterans without a PTSD diagnosis would have been ideal. Furthermore, the clinical sample was characterized by a long course since trauma of significant physical health problems, high psychiatric co-morbidity and high medication intake. Second, in addressing these sample confounds, statistical analysis covarying for IQ, anxiety, depression and alcohol found small effect sizes. Future research would benefit from more closely matched and larger sample sizes to explore the significance of OIDs in this clinical population. Finally, some aspects of the OI assessment also warrant refinement. Whilst participants were directed to alert the researcher to problems with detection, no formal olfactory detection assessment was conducted. The segregation of odors into nominal categories based on normative data may have also introduced some degree of error through this discretization.

As orbital prefrontal dysfunction has been implicated in several psychiatric disorders (see Brewer et al. 2006 for review), further research comparing OIDs across disorders is required to explore the implications for orbitofrontal function. For example, OIDs may be attributed to particular impairment in emotion modulation, assimilation of intero- and extero-receptive information, or perceptual response biases; these may be common to diverse clinical populations, or they may reflect a more general impairment OFC dysfunction. The methodology and sample limitations of this current study make it difficult to speculate how useful OI tests may be in delineating the complex functioning of the OFC. Employment of more customized OI tests in neuroimaging paradigms is required. If the predictive validity of OI tests can further be supported, such tasks have the potential to be an efficient, cost-effective and non-invasive method of identifying individuals 
who may be more susceptible to particular psychiatric outcomes such as aggression and impulsivity following war trauma, thereby allowing the focus of resources to be weighted towards assisting such individuals to re-assimilate into civilian life.

\section{Acknowledgments}

Associate Professor Brewer was supported by the National Health and Medical Research Council of Australia, Program Grant (ID 350241) and the Colonial Foundation. The authors gratefully acknowledge Dr Murat Yucel and colleagues for permitting the use of their Go/No-Go neurocognitive task, and Jeromy Anglim for his support in statistical analysis. We also thank all individuals who generously gave of their time to participate in this research, and the team at the Austin Hospital Veterans Psychiatry unit for aiding in recruitment.

\section{Declaration of Interest}

None.

\section{References}

Anderson AK, Christoff K, Stappen I, Panitz D, Ghahremani DG, Glover G, Gabrieli JD, Sobel N (2003). Dissociated neural representations of intensity and valence in human olfaction. Nature and Neuroscience 6, 196-202.

Babor T, Fuente J, Saunders J, Grant M (1989). The Alcohol Use Disorder Identification Test: Guidelines for Use in Primary Health Care. World Health Organisation, Division of Mental Health: Geneva.

Begic D, Jokic-Begic N (2001). Aggressive behavior in combat veterans with post-traumatic stress disorder. Military Medicine 166, 671-676.

Bennett-Levy J (1984). Determinants of performance on the Rey-Osterrieth Complex Figure Test: an analysis, and a new technique for single-case assessment. British Journal of Clinical Psychology 23, 109-119.

Best M, Williams JM, Coccaro EF (2002). Evidence for a dysfunctional prefrontal circuit in patients with an impulsive aggressive disorder. Proceedings of the National Academy of Sciences USA 99, 8448-8453.

Blair RJ (2004). The roles of orbital frontal cortex in the modulation of antisocial behavior. Brain $\mathcal{E}$ Cognition 55, 198-208.

Borkowoski JG, Benton AL, Spreen O (1967). Verbal fluency and brain damage. Neuropsychologia 5, 135-140.

Brewer WJ, Wood SJ, DeLuca C, Pantelis C (2006). Models of olfaction for exploring neurodevelopment. In Olfaction and the Brain (ed. W. J. Brewer, D. Castle and C. Pantelis). Cambridge University Press: New York.

Buss AH, Perry M (1992). The Aggression Questionnaire. Journal of Personality \& Social Psychology 63, 452-459.

Chemtob CM, Hamada RS, Roitblat HL, Muraoka MY (1994). Anger, impulsivity, and anger control in combat-related posttraumatic stress disorder. Journal of Consulting \& Clinical Psychology 62, 827-832.

Cohen J (1988). Statistical Power Analysis for the Behavioral Sciences, 2nd edn. Lawrence Earlbaum Associates: Hillsdale, NJ.

Critchley HD, Rolls ET (1996). Olfactory neuronal responses in the primate orbitofrontal cortex: Analysis in an olfactory discrimination task. Journal of Neurophysiology 75, 1659-1672.

Davidson RJ, Putnam KM, Larson CL (2000). Dysfunction in the neural circuitry of emotion regulation - a possible prelude to violence. Science 289, 591-594.

de Araujo IET, Rolls ET, Kringelbach ML, McGlone F, Phillips N (2003). Taste-olfactory convergence, and the representation of the pleasantness of flavour, in the human brain. European Journal of Neuroscience 18, 2059-2068.

Doty RL, Shaman P, Dann M (1984). Development of the University of Pennsylvania Smell Identification Test: a standardized microencapsulated test of olfactory function. Physiology E Behavior 32, 489-502.

Eysenck SB, Pearson PR, Easting G, Allsopp J (1985). Age norms for impulsiveness, venturesomeness and empathy in adults. Personality and Individual Differences 6, 613-610.

Hamann S (2003). Nosing in on the emotional brain. Nature Neuroscience 6, 106-108.

Joseph S, Dalgleish T, Thrasher S, Yule W (1997). Impulsivity and post-traumatic stress. Personality $\mathcal{E}$ Individual Differences 22, 279-281.

Kessler RC, Sonnega A, Bromet E, Hughes M, Nelson CB (1995). Posttraumatic stress disorder in the National Comorbidity Survey. Archives of General Psychiatry 52, 1048-1060.

Kline NA, Rausch JL (1985). Olfactory precipitants of flashbacks in posttraumatic stress disorder. Journal of Clinical Psychiatry 46, 383-384.

Kotler M, Iancu I, Efroni R, Amir M (2001). Anger, impulsivity, social support, and suicide risk in patients with posttraumatic stress disorder. Journal of Nervous $\mathcal{E}$ Mental Disease 189, 162-167.

Luria AR (ed.) (1969). Frontal Lobe Syndromes. Elsevier: New York.

Mackay-Sim A, Doty RL (2001). The University of Pennsylvania smell identification test: Normative adjustment for Australian subjects. Australian Journal of Oto-Laryngology 4, 174.

Martzke JS, Kopala LC, Good KP (1997). Olfactory dysfunction in neuropsychological disorders: review and methodological considerations. Biolological Psychiatry 42, 721-732.

Nelson HE, O'Donnell A (1978). Dementia: the estimation of premorbid intelligence levels using the National Adult Reading Test. Cortex 14, 234-244.

O'Donnell ML, Creamer M, Pattison P (2004). Posttraumatic stress disorder and depression following trauma: Understanding comorbidity. American Journal of Psychiatry 161, 1390-1396.

Oozthuizen F, Wegener G, Harvey BH (2005). Nitric oxide as inflammatory mediator in posttraumatic stress disorder (PTSD): evidence from an animal model. Neuropsychiatric Disease and Treatment 1, 109-123. 
Page MC, Braver SL, MacKinnon DP (2003). Levine's Guide to SPSS for analysis of variance, 2nd edn. Erlbaum: Mahwah, NJ.

Pietrini P, Guazzelli M, Basso G, Jaffe K, Grafman J (2000). Neural correlates of imaginal aggressive behavior assessed by positron emission tomography in healthy subjects. American Journal of Psychiatry 157, 1772-1781.

Rey A (1941). L'examen psychologique dans les cas d'encephalopathie traumatique. Archives de Psychologie 28.

Rolls ET (1998). The Orbitofrontal Cortex. Oxford University Press: London.

Rolls ET (2000). The orbitofrontal cortex and reward. Cerebral Cortex (Special: The Mysterious Orbitofrontal Cortex) 10, 284-294.

Rolls ET, Kringelbach ML, de Araujo IET (2003). Different representations of pleasant and unpleasant odours in the human brain. European Journal of Neuroscience 18, 695-703.

Seguin JR (2004). Neurocognitive elements of antisocial behavior: relevance of an orbitofrontal cortex account. Brain \& Cognition 55, 185-197.

Stanley B, Molcho A, Stanley M, Winchel R, Gameroff MJ, Parsons B, Mann JJ (2000). Association of aggressive behavior with altered serotonergic function in patients who are not suicidal [see comment]. American Journal of Psychiatry 157, 609-614.

Taylor EM (1959). Psychological Appraisal of Children with Cerebral Deficits. Harvard University Press: Cambridge, MA.

Turner SM, Beidel DC, Frueh BC (2005). Multicomponent behavioral treatment for chronic combat-related posttraumatic stress disorder: trauma management therapy. Behavior Modification. Special: Beyond Exposure for
Posttraumatic Stress Disorder Symptoms: Broad-Spectrum PTSD Treatment Strategies 29, 39-69.

Vasterling JJ, Brailey K, Sutker PB (2000). Olfactory identification in combat-related posttraumatic stress disorder. Journal of Traumatic Stress 13, 241-253.

Vasterling JJ, Brailey K, Tomlin H, Rice J, Sutker PB (2003). Olfactory functioning in Gulf War-era veterans: relationships to war-zone duty, self-reported hazards exposures, and psychological distress. Journal of the International Neuropsychological Society 9, 407-418.

Vermetten E, Bremner JD (2002). Circuits and systems in stress. I. Preclinical studies. Depression \& Anxiety 15, 126-147.

Vermetten E, Bremner JD (2003). Olfaction as a traumatic reminder in posttraumatic stress disorder: Case reports and review. Journal of Clinical Psychiatry 64, 202-207.

Weathers FW, Litz B, Herman D, Huska J, Keane T (1993). The PTSD Checklist (PCL): Reliability, Validity, and Diagnostic Utility. Paper presented at the Annual Convention of the International Society for Traumatic Stress Studies, San Antonio, TX, USA.

Wechsler D (1987). Wechsler Memory Scale - Revised Manuel. The Psychological Corporation: San Antonio.

Zatorre RJ, Jones-Gotman M, Evans AC, Meyer E (1992). Functional localization and lateralization of human olfactory cortex. Nature 360, 339-340.

Zatorre RJ, Jones-Gotman M, Rouby C (2000). Neural mechanisms involved in odor pleasantness and intensity judgments. Neuroreport 11, 2711-2716.

Zigmond AS, Snaith RP (1983). The Hospital Anxiety and Depression Scale. Acta Psychiatrica Scandinavica 67, 361-370. 


\section{University Library}

\section{- M M I N E R VA \\ A gateway to Melbourne's research publications}

Minerva Access is the Institutional Repository of The University of Melbourne

Author/s:

Dileo, JF;Brewer, WJ;Hopwood, M;Anderson, V;Creamer, M

Title:

Olfactory identification dysfunction, aggression and impulsivity in war veterans with posttraumatic stress disorder

Date:

2008-04-01

Citation:

Dileo, J. F., Brewer, W. J., Hopwood, M., Anderson, V. \& Creamer, M. (2008). Olfactory identification dysfunction, aggression and impulsivity in war veterans with post-traumatic stress disorder. PSYCHOLOGICAL MEDICINE, 38 (4), pp.523-531. https://doi.org/10.1017/ S0033291707001456.

Publication Status:

Published

Persistent Link:

http://hdl.handle.net/11343/33011 